\title{
Ptychographic optical coherence tomography
}

\author{
Mengq Du, ${ }^{1,2, *}$ (1) Lars Loetgering, ${ }^{1,2}$ [1) KJeld S. E. Eikema, ${ }^{1,2}$ and Stefan Witte ${ }^{1,2,3}$ [1] \\ ${ }^{1}$ Advanced Research Center for Nanolithography, Science Park 106, 1098 XG Amsterdam, The Netherlands \\ ${ }^{2}$ Vrije Universiteit, De Boelelaan 1105, 1081 HV Amsterdam, The Netherlands \\ ${ }^{3}$ e-mail:witte@arcnl.nl \\ *Corresponding author:m.du@arcnl.nl
}

Received 30 November 2020; revised 29 January 2021; accepted 10 February 2021; posted 12 February 2021 (Doc. ID 416144); published 10 March 2021

\begin{abstract}
Ptychography is a robust computational imaging technique that can reconstruct complex light fields beyond conventional hardware limits. However, for many wide-field computational imaging techniques, including ptychography, depth sectioning remains a challenge. Here we demonstrate a high-resolution three-dimensional (3D) computational imaging approach, which combines ptychography with spectral-domain imaging, inspired by optical coherence tomography (OCT). This results in a flexible imaging system with the main advantages of OCT, such as depth-sectioning without sample rotation, decoupling of transverse and axial resolution, and a high axial resolution only determined by the source bandwidth. The interferometric reference needed in OCT is replaced by computational methods, simplifying hardware requirements. As ptychography is capable of deconvolving the illumination contributions in the observed signal, speckle-free images are obtained. We demonstrate the capabilities of ptychographic optical coherence tomography (POCT) by imaging an axially discrete lithographic structure and an axially continuous mouse brain sample. () 2021 Optical Society of America under the terms of the OSA Open Access Publishing Agreement
\end{abstract}

https://doi.org/10.1364/OL.416144

Ptychography $[1,2]$ has been successfully applied for twodimensional (2D) imaging using various radiation sources, e.g., optical, $\mathrm{x}$ ray, and electron sources [3-6], and various methods have been investigated to extend its applicability to three dimensions (3D). The 2D ptychographic forward model assumes a thin object such that the interaction between the illumination and object can be factorized into a simple multiplication [5]. For thicker samples that do not satisfy this multiplication condition, multislice ptychography [7-11] computationally divides a sample into a series of thin slices, applying the $2 \mathrm{D}$ forward model from each slice to the next to reconstruct a $3 \mathrm{D}$ image. However, the depth resolution of multislice ptychography is limited to the order of the depth-of-field (DoF) of the imaging system. Another approach to extend conventional ptychography is to combine it with other 3D imaging modalities. In the $\mathrm{x}$ ray regime, techniques termed ptychographic tomography (PT) [12-14] and ptychographic laminography
[15] reconstruct a $3 \mathrm{D}$ image from $2 \mathrm{D}$ projections at different object viewing angles. Recently, multislice ptychographic tomography (MSPT) [16] has been introduced to extend the imaging depth of the technique while preserving the depth resolution. But in general, a drawback of tomographic techniques is the requirement for dense, time consuming angular scans. In the optical regime, optical coherence tomography (OCT) has become a prominent 3D imaging method [17-19]. OCT obtains 3D information by measuring interference between light backscattered from an object and an external reference by taking advantage of the low temporal coherence of a broadband light source. OCT has also been extended and combined with other computational imaging techniques to account for refractive distortion [20] and diffraction effects inside the imaged $3 \mathrm{D}$ volume [21-23]. An OCT system requires the use of a stable interferometric reference arm to encode temporal phase. Furthermore, OCT suffers from speckle noise [19] intrinsic to most coherent imaging methods.

Here we present a new technique that enables high-resolution $3 \mathrm{D}$ imaging in a reflection geometry, without the need for object rotation or interferometry, by combining ptychography with swept-source OCT. We demonstrate this ptychographic optical coherence tomography (POCT) technique using a nearinfrared swept-source laser in a lensless ptychography setup, where a fast wavelength scan is performed at each transverse scan position. Similar to OCT, the axial resolution in POCT is decoupled from the lateral resolution and is only limited by the source spectral bandwidth. This feature enables fine depth sectioning, not limited by the DoF of the imaging setup that would limit a multislice ptychography approach. The electronically controlled wavelength scan offers a speed advantage over angular scans needed in tomographic approaches. Furthermore, by using ptychography's ability to retrieve the complex-valued fields of both the probe beam and the object, POCT provides clean tomographic reconstructions free of speckle artefacts that are common in OCT. As the phase is retrieved through computational methods, the interferometric reference arm can be eliminated from the setup, resulting in a simple, compact, and robust layout. We show POCT reconstructions of both an axially discrete two-layer lithographic sample and an axially continuous mouse brain sample.

Figure 1 shows the schematic of the POCT experimental setup where a swept-source is used in a reflection ptychography 


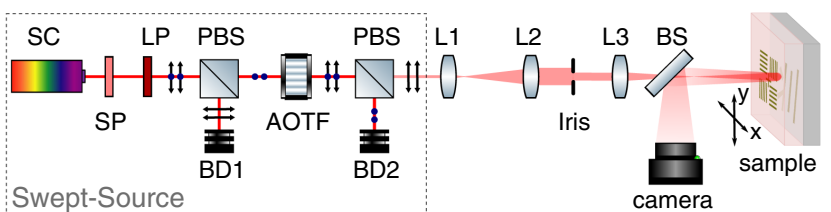

Fig. 1. POCT experimental setup. The swept-source section consists of a supercontinuum laser (SC), short pass (SP, $1000 \mathrm{~nm}$ ) and long pass (LP, $700 \mathrm{~nm}$ ) filters, polarizing beam splitters (PBS), and an acousto-optic tunable filter (AOTF, Gooch \& Housego, TF950-5001-2-GH96). BD1,2, beam dumps. Lenses L1 $\left(f_{1}=50 \mathrm{~mm}\right)$ and L2 $\left(f_{2}=150 \mathrm{~mm}\right)$ are used to expand the beam. An iris is imaged onto the sample surface by the lens $\mathrm{L} 3\left(f_{3}=50 \mathrm{~mm}\right)$. The object is mounted on an encoded 2D translation stage $(2 \times$ Smaract SLC-1770-D-S, 46 $\mathrm{mm}$ travel range, $70 \mathrm{~nm}$ repeatability). The backscattered light from the sample is directed by a beam splitter (BS) to a CCD camera (AVT prosilica GX1920, 14 bit, pixel size $4.54 \mu \mathrm{m})$.

configuration. Details of the swept-source system are described in [24]. In the reflection ptychography part, the sample is translated relative to the stationary probe beam with overlapping areas by a 2D motorized stage. A Fermat's spiral pattern [25] is used as the scan grid. At each transverse scan position, an equidistant frequency scan is performed, and monochromatic $(\lambda / \Delta \lambda>1000)$ backscattered intensity patterns are recorded by the camera at each frequency. Lenses L1, L2, L3 and the iris are used to create and control a localized probe for ptychography.

After the data acquisition, single-slice ptychographic reconstructions are performed at each wavelength. Theoretically, the multiplicative approximation in single-slice ptychography holds [26] when the sample thickness $T$ satisfies $T \leq 8(\Delta r)^{2} / \lambda$, where $\lambda$ is the wavelength and $\Delta r=\lambda / 2 \mathrm{NA}$ is the lateral halfpitch resolution. Under the multiplicative approximation, single-slice ptychography offers quantitative object reconstruction, with which high-resolution surface profilometry has been successfully demonstrated [3,27]. By measuring semi-transparent samples and using multiple-wavelength measurements, POCT aims at volumetric imaging.

Our data analysis pipeline is summarized in Fig. 2. At the starting wavelength, we use an auto-focus algorithm specifically tailored to ptychography [28] to calibrate the sample-detector distance. Using this calibration, a momentum-accelerated ePIE algorithm [29,30] is used to reconstructed the object and probe. Thanks to the small step size in the wavelength scan, the previously reconstructed probe and object can be used as initial guesses for the following reconstruction to speed up the convergence rate. In this way, ptychographic reconstructions are performed wavelength by wavelength.

Although quantitative object reconstructions are obtained at each wavelength, initially they appear uncorrelated because of several reasons. First, the reconstructed object pixel size is wavelength-dependent when using Fraunhofer propagation [31]. Second, the absolute lateral object position is one of the ambiguities of ptychography, causing independent reconstructions to contain arbitrary spatial shifts as indicated in Fig. 2. Last, there is a random constant phase term for each object reconstruction due to the non-uniqueness of a constant phase offset in ptychography. For lateral alignment, all estimated objects are scaled and linearly interpolated to the reconstruction with the smallest pixel size, and then registered with sub-pixel precision [32]. The phase relation between the different-wavelength images can be determined from the object

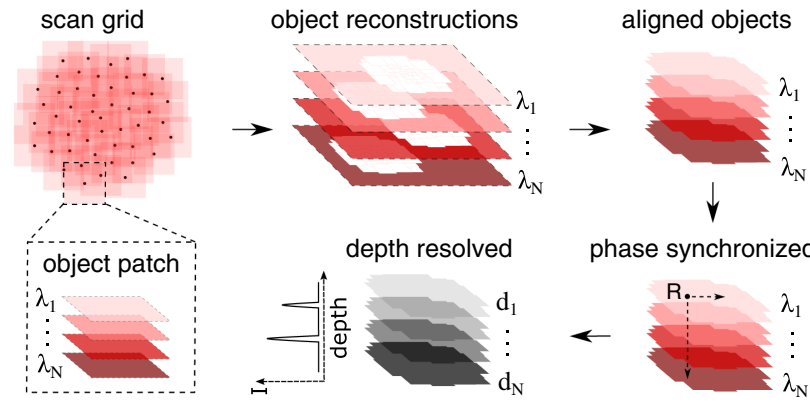

Fig. 2. POCT data analysis pipeline. Single-slice ptychographic reconstructions are performed for each wavelength, and then all object reconstructions are aligned by scaling and registration, and phase synchronized using a synthetic reference signal. A final Fourier transform reveals the depth distribution.

itself, thereby replacing the external interferometric reference surface in swept-source OCT, provided the signal is dominated by a single depth reflection at one object location. In contrast to common-path OCT [33], no flat reference layer is required as phase shifts in one layer are identified by the ptychographic reconstruction upon phase synchronization at a single object location (pixel). Once a reference position is chosen, we synchronize the constant phase offsets of all wavelengths at this position. Finally, a 1D Fourier transform along the wavelength axis reveals the depth information of the object. Given by the Fourier transform relation, the optical sectioning resolution is

$$
\Delta z_{\mathrm{opt}}=\frac{\lambda_{1} \lambda_{N}}{\left|\lambda_{1}-\lambda_{N}\right|} .
$$

Taking into account the refractive index $n$ of the object and the double pass in the reflection geometry, the physical depth resolution is given by $\Delta z_{\text {phy }}=\Delta z_{\mathrm{opt}} / 2 n$.

As a first demonstration of POCT, a custom-made two-layer sample has been measured using $N=51$ wavelengths ranging from $708.8 \mathrm{~nm}$ to $802.8 \mathrm{~nm}$ at $M=201$ transverse scan positions. The sample consists of semi-transparent metal structures $(\sim 50 \mathrm{~nm}$ thick) axially separated by a layer of transparent polymer. Fabrication details of the sample can be found in [24]. A $3 \mathrm{D}$ representation of the sample is shown as part of the setup schematic in Fig. 1, and Fig. 3(a) shows the optical microscope image of the sample. The sample-detector distance calibrated by zPIE [28] is $38.6 \mathrm{~mm}$. At the shortest wavelength of 708.8 $\mathrm{nm}$ and with the detection NA of 0.085 , the lateral resolution is $\Delta r=\lambda_{\max } / 2 \mathrm{NA}=4.7 \mu \mathrm{m}$, and the DoF is $240 \mu \mathrm{m}$. The optical sectioning resolution $\Delta z_{\text {opt }}$ based on Eq. (1) is $6 \mu \mathrm{m}$, resulting in a depth resolution of $2 \mu \mathrm{m}$, an improvement over conventional ptychography by 2 orders of magnitude. See Supplement 1 for intermediate results. The resolved tomograms of the top and the bottom layers are shown in Figs. 3(b) and 3(c), respectively. Figure 3(d) shows three depth plots at three locations marked in Figs. 3(b) and 3(c), respectively. The phase synchronization was performed at location 1 . The width of the peaks shows that the expected depth resolution of $2 \mu \mathrm{m}$ is indeed achieved. We do observe a slight broadening of the peak at location 2 , which is caused by strongly dispersive spacer layer as also observed in OCT measurements [24]. Phase synchronization at location 2 would have led to a spectrum-limited resolution at this depth, and more advanced numerical dispersion compensation should enable depth-independent resolution. Because 

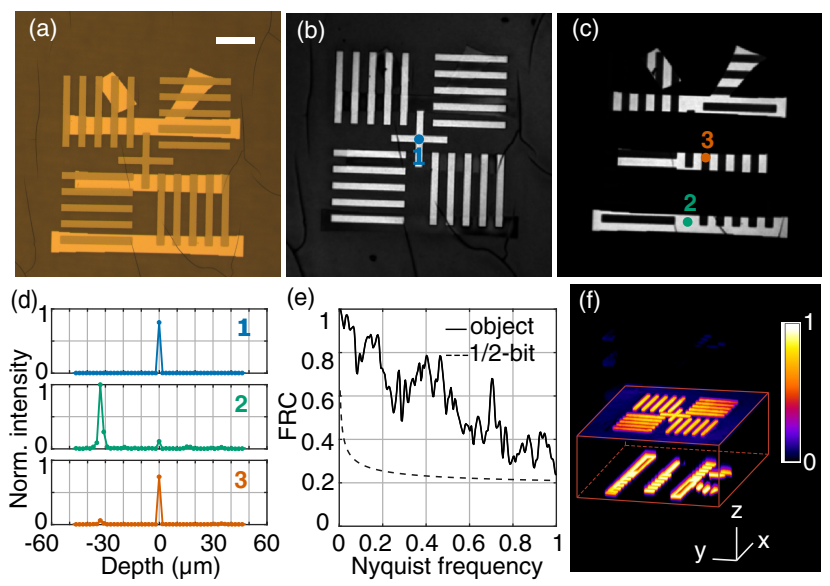

Fig. 3. (a) Bright-field optical microscope image of the sample. Scale bar is $200 \mu \mathrm{m}$. (b), (c) Reconstructed tomograms of the top and bottom layers, respectively. (d) Depth response at three locations marked in (b), (c). (e) Solid line, Fourier ring correlation (FRC) between object intensities reconstructed at the shortest $(708.8 \mathrm{~nm})$ and longest $(802.8 \mathrm{~nm})$ wavelengths. Dashed line, the $1 / 2$ bit resolution curve. (f) $3 \mathrm{D}$ rendering of the reconstruction, showing the layer separation. The colorscale shows the normalized intensity (linear scale). The distance in the $z$ direction is increased by a factor of 4 compared to the $x$ and $y$ directions. A multi-angle projection of this $3 \mathrm{D}$ reconstruction is shown in Visualization 1.

phase retrieval provides complex-valued information rather than interferometric intensity measurements, the depth sections in Fig. 3(d) largely suppress any autocorrelation artefacts or complex conjugate signals common to spectral-domain OCT. The lateral resolution is evaluated by calculating the Fourier ring correlation (FRC) [34] of two object intensities reconstructed from the shortest $(708.8 \mathrm{~nm})$ and longest wavelengths $(803.8$ $\mathrm{nm}$ ), and the $1 / 2$ bit resolution criteria are used. In Fig. 3(e), the object correlation curve (solid line) is above the $1 / 2$ bit threshold (dashed line) across the detected spatial frequency spectrum, meaning that the two independent reconstructions are consistent up to the Nyquist frequency. Thus, the measured half-period lateral resolution in our lensless setup is confirmed to be at the diffraction limit of $4.7 \mu \mathrm{m}$. A 3D rendering of the sample is shown in Fig. 3(f). As depicted in Fig. 1, the top layer is the metal/air or polymer/air interface, and the bottom layer has metal structures at the polymer/glass interface with matching refractive index. A 3D visualization video is available online (Visualization 1).

In a second experiment, a cryo-cut unstained slice of cerebellar mouse brain tissue with a nominal thickness of $40 \mu \mathrm{m}$ has been measured using POCT. This mouse brain sample has continuous axial structures. A low-resolution, bright-field, reflection optical microscope image of the sample is shown in Fig. 4(a). A high-NA objective is used in the microscope to focus on the top and the bottom layer of the sample, images of which are shown in Figs. 4(b) and 4(c). In the experiment, the sample is measured with $N=49$ wavelengths ranging from $708.8 \mathrm{~nm}$ to $851.3 \mathrm{~nm}$ at $M=201 \mathrm{scan}$ positions. The optical sectional resolution $\Delta z_{\mathrm{opt}}$ is $4.3 \mu \mathrm{m}$. The calibrated propagation distance from the object to the detector is $25.4 \mathrm{~mm}$, resulting in a detection NA of 0.12 , a lateral half-period resolution of $3 \mu \mathrm{m}$, and a DoF of $106 \mu \mathrm{m}$. Figure 4(d) shows the reconstructed tomogram of the deepest layer of the mouse brain tissue, where

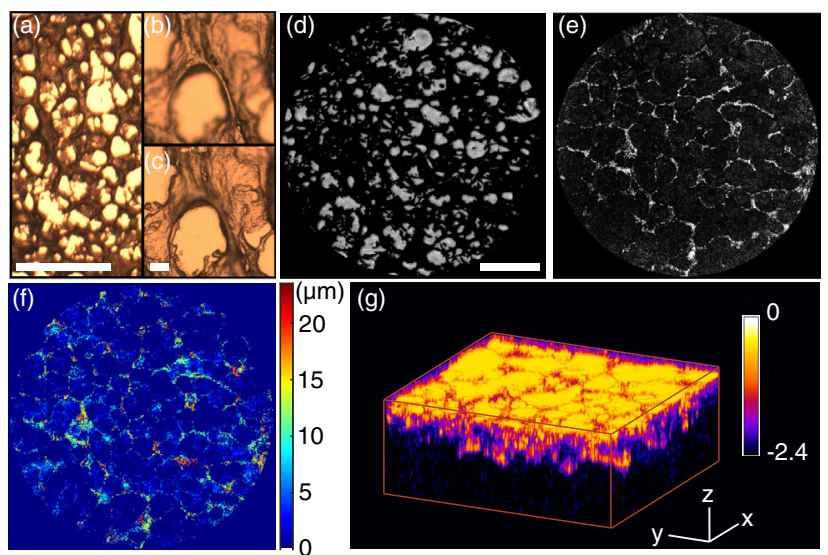

Fig. 4. (a) Low-NA $(0.13 / 5 x)$ optical microscope image of the mouse brain sample. Scale bar is $500 \mu \mathrm{m}$. (b), (c) High-NA $(0.45 / 50 \mathrm{x})$ optical microscope images of the mouse brain sample focused at the top and bottom structures, respectively. Scale bar is $20 \mu \mathrm{m}$, shared between (b) and (c). (d) Reconstructed tomogram of the bottom layer of the mouse brain sample [the air/glass (bright) or the mouse brain/glass (dark) interface]. Scale bar is $500 \mu \mathrm{m}$, shared among (d)-(f). (e) Reconstructed tomogram of the top layer of the sample (the air/mouse brain interface). (f) Color-coded height map of the mouse brain sample. (g) 3D rendering of part of the reconstructed mouse brain. The colorscale shows the logarithm of the normalized reflectivity. A magnification factor 4 was applied in the $z$ direction. A rotating projection of this $3 \mathrm{D}$ reconstruction is shown in Visualization 2.

the bright areas indicate the air/glass substrate interface and the dark areas indicate the brain/glass interface. A tomogram of an upper layer of the sample is shown in Fig. 4(e), where the bright areas are ridges of the mouse brain tissue that correspond to the sharp structures in Fig. 4(b). Using an averaged value of $n=1.38$ for the refractive index of mouse brain tissue across the full wavelength range [35], we obtain a value for the thickness of the mouse brain of $20 \mu \mathrm{m}$ with a depth resolution of $1.6 \mu \mathrm{m}$. Figure 4(f) shows a color-coded height plot of the reconstructed sample, where for each pixel the height is chosen at the maximum intensity location along the $z$ direction. Figure $4(\mathrm{~g})$ shows a $3 \mathrm{D}$ rendering of the reconstructed mouse brain sample. See Visualization 2 for a 3 D rotating projection.

With the two-layer and mouse brain samples, we demonstrate that POCT successfully performs depth-resolved imaging, where the lateral and axial resolutions are decoupled. This shows that POCT has a great potential for $3 \mathrm{D}$ high-resolution imaging. Compared to OCT, POCT does not rely on an external reference, and it deconvolves the illumination from the object reconstruction. Therefore, POCT does not suffer from reference- and illumination-induced artefacts. Additionally, the current setup only contains a beam splitter between the object and the camera; thus, the reconstruction results [Figs. 3(b) and 3(c) and Figs. 4(d) and 4(e)] are almost free of typical coherent imaging artefacts ("speckle") caused by imperfections in optical systems.

One advantage of POCT over CT-based 3D imaging techniques $[12-14,16]$ is speed. First, wavelength scanning is faster than mechanical stage rotation, as our acousto-optic scanning method enables sub-ms wavelength switching times, and fast cameras achieve multi-kHz update rates. Second, computational complexity is reduced, as POCT only requires a series 
of $2 \mathrm{D}$ reconstructions plus a phase synchronisation step, and the correlation between different-wavelength images provides a strong initial guess for both object and probe after a first reconstruction at a single wavelength, ensuring fast convergence. Furthermore, no image stitching algorithms are needed. Lastly, as the phase synchronization is achieved numerically, no phase-stable optical path is needed. In addition to the present swept-source implementation of POCT, the concept can be extended to other methods in which wavelength-resolved diffraction data is measured, such as Fourier-transform-based diffractive imaging [36,37] or computational time-domain OCT [24].

Going beyond the present proof-of-concept, various improvements can still be envisaged. One clear limitation of POCT is the absence of coherent amplification by a strong reference wave. We, therefore, envision the main applications of POCT in material science and lithographic device inspection, where photon flux on sample is not the limiting factor, or in situations where the generation of a reference beam is challenging such as with extreme ultraviolet radiation. While in the lensless geometry the NA is limited by the sample-detector distance and detector size, the use of high-NA optics can yield a significant transverse resolution improvement. In addition, the axial imaging range is currently limited to the single-layer projection limit in ptychography, which is typically in the order of tens or hundreds microns at optical wavelengths. In principle, multislice ptychography [8-11] can be applied to extend this limit. Moreover, the current POCT implementation has not taken sample dispersion into account, which may be possible through adaptive optics [23]. Finally, although single-layer ptychography could handle multiple scattering to a certain degree, more sophisticated forward modeling in ptychography is required to apply POCT on highly scattering samples in reflection.

In conclusion, we have introduced POCT as a new concept for high-resolution 3D optical imaging. Further improvements to this new imaging modality are clearly possible, and we believe that the POCT concept has great potential as a simple yet robust 3D imaging method, especially on wafer-based planar nanostructures commonly found in modern nanotechnology.

Funding. Nederlandse Organisatie voor Wetenschappelijk Onderzoek (13934); European Research Council (637476).

Acknowledgment. We thank Karl Schilling (Uni. Bonn) for providing mouse brain samples.

Disclosures. The authors declare no conflicts of interest.

Supplemental document. See Supplement 1 for supporting content.

\section{REFERENCES}

1. J. M. Rodenburg and H. M. L. Faulkner, Appl. Phys. Lett.85, 4795 (2004).

2. J. Rodenburg and A. Maiden, in Springer Handbook of Microscopy, P. Hawkes and J. Spence, eds. (Springer, 2019), Chap. 17, pp. 819904.

3. D. Claus, D. J. Robinson, D. G. Chetwynd, Y. Shuo, W. T. Pike, J. J. De Jesus Toriz-Garcia, and J. M. Rodenburg, J. Opt. 15, 035702 (2013).

4. D. F. Gardner, M. Tanksalvala, E. R. Shanblatt, X. Zhang, B. R. Galloway, C. L. Porter, R. Karl, C. Bevis, D. E. Adams, H. C. Kapteyn, M. M. Murnane, and G. F. Mancini, Nat. Photonics11, 259 (2017).
5. P. Thibault, M. Dierolf, A. Menzel, O. Bunk, C. David, and F. Pfeiffer, Science 321, 379 (2008).

6. Y. Jiang, Z. Chen, Y. Han, P. Deb, H. Gao, S. Xie, P. Purohit, M. W. Tate, J. Park, S. M. Gruner, V. Elser, and D. A. Muller, Nature 559, 343 (2018).

7. A. M. Maiden, M. J. Humphry, and J. M. Rodenburg, J. Opt. Soc. Am. A29, 1606 (2012).

8. T. M. Godden, R. Suman, M. J. Humphry, J. M. Rodenburg, and A. M. Maiden, Opt. Express22, 12513 (2014).

9. E. H. R. Tsai, I. Usov, A. Diaz, A. Menzel, and M. Guizar-Sicairos, Opt. Express24, 29089 (2016).

10. A. Suzuki, S. Furutaku, K. Shimomura, K. Yamauchi, Y. Kohmura, T. Ishikawa, and Y. Takahashi, Phys. Rev. Lett.112, 053903 (2014).

11. S. Gao, P. Wang, F. Zhang, G. T. Martinez, P. D. Nellist, X. Pan, and A. I. Kirkland, Nat. Commun.8, 163 (2017).

12. M. Dierolf, A. Menzel, P. Thibault, P. Schneider, C. M. Kewish, R. Wepf, O. Bunk, and F. Pfeiffer, Nature 467, 436 (2010).

13. M. Holler, A. Diaz, M. Guizar-Sicairos, P. Karvinen, E. Färm, E. Härkönen, M. Ritala, A. Menzel, J. Raabe, and O. Bunk, Sci. Rep. 4, 3857 (2014).

14. M. Holler, M. Guizar-Sicairos, E. H. Tsai, R. Dinapoli, E. Müller, O. Bunk, J. Raabe, and G. Aeppli, Nature 543, 402 (2017).

15. M. Holler, M. Odstrcil, M. Guizar-Sicairos, M. Lebugle, E. Müller, S. Finizio, G. Tinti, C. David, J. Zusman, W. Unglaub, O. Bunk, J. Raabe, A. F. J. Levi, and G. Aeppli, Nat. Electron.2, 464 (2019).

16. P. Li and A. Maiden, Sci. Rep. 8, 2049 (2018).

17. D. Huang, E. A. Swanson, C. P. Lin, J. S. Schuman, W. G. Stinson, W. Chang, M. R. Hee, T. Flotte, K. Gregory, and C. A. Puliafito, Science 254, 1178 (1991)

18. S. A. Boppart, B. E. Bouma, C. Pitris, J. F. Southern, M. E. Brezinski, and J. G. Fujimoto, Nat. Med. 4, 861 (1998).

19. J. F. de Boer, R. Leitgeb, and M. Wojtkowski, Biomed. Opt. Express8, 3248 (2017).

20. K. C. Zhou, R. Qian, S. Degan, S. Farsiu, and J. A. Izatt, Nat. Photonics13, 794 (2019).

21. T. S. Ralston, D. L. Marks, P. S. Carney, and S. A. Boppart, Nat. Phys.3, 129 (2007).

22. N. D. Shemonski, F. A. South, Y. Z. Liu, S. G. Adie, P. S. Carney, and S. A. Boppart, Nat. Photonics9, 440 (2015).

23. Y.-Z. Liu, F. A. South, Y. Xu, P. S. Carney, and S. A. Boppart, Biomed. Opt. Express8, 1549 (2017).

24. M. Du, K. S. E. Eikema, and S. Witte, OSA Contin.2, 3141 (2019).

25. X. Huang, H. Yan, R. Harder, Y. Hwu, I. K. Robinson, and Y. S. Chu, Opt. Express22, 12634 (2014).

26. J. M. Rodenburg and R. H. T. Bates, Philos. Trans. R. Soc. London A 339, 521 (1992).

27. M. D. Seaberg, B. Zhang, D. F. Gardner, E. R. Shanblatt, M. M. Murnane, H. C. Kapteyn, and D. E. Adams, Optica 1, 39 (2014).

28. L. Loetgering, M. Du, K. S. E. Eikema, and S. Witte, Opt. Lett.45, 2030 (2020).

29. A. M. Maiden and J. M. Rodenburg, Ultramicroscopy109, 1256 (2009).

30. A. Maiden, D. Johnson, and P. Li, Optica 4, 736 (2017).

31. J. W. Goodman, Introduction to Fourier Optics (Roberts and Company, 2005).

32. M. Guizar-Sicairos, S. T. Thurman, and J. R. Fienup, Opt. Lett.33, 156 (2008).

33. M. V. Sarunic, S. Weinberg, and J. A. Izatt, Opt. Lett.31, 1462 (2006).

34. M. Van Heel and M. Schatz, J. Struct. Biol.151, 250 (2005).

35. J. Binding, J. B. Arous, J.-F. Léger, S. Gigan, C. Boccara, and L. Bourdieu, Opt. Express19, 4833 (2011).

36. S. Witte, V. T. Tenner, D. W. E. Noom, and K. S. E. Eikema, Light Sci. Appl.3, e163 (2014).

37. G. S. M. Jansen, D. Rudolf, L. Freisem, K. S. E. Eikema, and S. Witte, Optica 3,1122 (2016). 